TITLE:

\title{
Characteristics of the horizontal component of Rayleigh waves in multimode analysis of surface
} waves

\section{$\operatorname{AUTHOR}(\mathrm{S}):$}

Ikeda, Tatsunori; Matsuoka, Toshifumi; Tsuji, Takeshi; Nakayama, Toru

\section{CITATION:}

Ikeda, Tatsunori ...[et al]. Characteristics of the horizontal component of Rayleigh waves in multimode analysis of surface waves. GEOPHYSICS 2014, 80(1): EN1-EN11

\section{ISSUE DATE:}

2014-12-01

URL:

http://hdl.handle.net/2433/196863

\section{RIGHT:}

(C) 2014 Society of Exploration Geophysicists. Notice: be provided that use is subject to SEG terms of use and condition. 


\title{
Characteristics of the horizontal component of Rayleigh waves in multimode analysis of surface waves
}

\author{
Tatsunori Ikeda ${ }^{1}$, Toshifumi Matsuoka ${ }^{2}$, Takeshi Tsuji ${ }^{3}$, and Toru Nakayama ${ }^{4}$
}

\begin{abstract}
In surface-wave analysis, $\mathrm{S}$-wave velocity estimations can be improved by the use of higher modes of the surface waves. The vertical component of P-SV waves is commonly used to estimate multimode Rayleigh waves, although Rayleigh waves are also included in horizontal components of P-SV waves. To demonstrate the advantages of using the horizontal components of multimode Rayleigh waves, we investigated the characteristics of the horizontal and vertical components of Rayleigh waves. We conducted numerical modeling and field data analyses rather than a theoretical study for both components of Rayleigh waves. As a result of a simulation study, we found that the estimated higher modes have larger relative amplitudes in the vertical and horizontal components as the
\end{abstract}

source depth increases. In particular, higher-order modes were observed in the horizontal component data for an explosive source located at a greater depth. Similar phenomena were observed in the field data acquired by using a dynamite source at 15-m depth. Sensitivity analyses of dispersion curves to $\mathrm{S}$-wave velocity changes revealed that dispersion curves additionally estimated from the horizontal components can potentially improve S-wave velocity estimations. These results revealed that when the explosive source was buried at a greater depth, the horizontal components can complement Rayleigh waves estimated from the vertical components. Therefore, the combined use of the horizontal component data with the vertical component data would contribute to improving $\mathrm{S}$-wave velocity estimations, especially in the case of buried explosive source signal.

\section{INTRODUCTION}

The surface-wave method is a widely applied nondestructive method for estimating subsurface S-wave velocity structures, for example, for mapping bedrock (Miller et al., 1999), assessment of soil liquefaction potential (Lin et al., 2004), pavement structures (Ryden and Lowe, 2004), glaciers (Tsuji et al., 2012), and understanding fault geometry (Ikeda et al., 2013). Socco et al. (2010) provide a comprehensive review of the surface-wave analysis technique. In onshore seismic reflection surveys, surface waves are usually considered to be noise and are therefore eliminated. However, once surface waves can be obtained and properly analyzed, they can be used to estimate near-surface structures for static corrections (Strobbia et al., 2010; R. Askari, personal communication, 2013) and filtered from raw data (Strobbia et al., 2011).
Surface waves have different propagation velocities at different frequencies. The propagation mode with the slowest phase velocity is the fundamental mode, and all other propagation modes are referred to as higher modes. Most surface-wave analyses use the fundamental mode, but it is well known that higher modes gain in importance if a stiff layer either overlies a soft layer or is embedded between soft layers (e.g., Gucunski and Woods, 1992; Tokimatsu et al., 1992). The sensitivities of higher modes of surface waves differ from the fundamental mode sensitivity; when the wavelength is the same, higher modes are more sensitive to $S$-wave velocities in deeper layers compared with the fundamental mode (Xia et al., 2003). The sensitivities of higher modes cover a wider frequency band than the fundamental mode sensitivity (Luo et al., 2007). Therefore, multimode analysis of surface waves can potentially

\footnotetext{
Manuscript received by the Editor 14 January 2014; revised manuscript received 22 August 2014; published online 1 December 2014.

${ }^{1}$ Formerly Kyoto University, Department of Urban Management, Kyoto, Japan; presently Kyushu University, International Institute for Carbon-Neutral Energy Research (WPI-I2CNER), Fukuoka, Japan. E-mail: ikeda@i2cner.kyushu-u.ac.jp.

${ }^{2}$ Kyoto University, Department of Urban Management, Kyoto, Japan. E-mail: matsuoka.toshifumi.7x @ kyoto-u.ac.jp.

${ }^{3}$ Kyushu University, International Institute for Carbon-Neutral Energy Research (WPI-I2CNER), Fukuoka, Japan. E-mail: tsuji@i2cner.kyushu-u.ac.jp.

${ }^{4} J a p a n$ Petroleum Exploration Co., Ltd., Tokyo, Japan. E-mail: toru.nakayama@japex.co.jp.

(C) 2014 Society of Exploration Geophysicists. All rights reserved.
} 
improve the resolution of S-wave velocity estimations and allow the investigation depth to be increased.

Most previous studies have obtained Rayleigh-wave dispersion curves only from the vertical component of P-SV waves. Very few studies have investigated the horizontal component of Rayleigh waves, which are included in the horizontal (radial) component of P-SV waves. Dal Moro and Ferigo (2011) show, however, that the multimode components of Rayleigh waves estimated from the horizontal component data have a different energy distribution compared with those estimated from the vertical component data. Therefore, they point out that jointly analyzing both components has a high potential for providing robust spectra interpretation. Because Rayleigh waves have common theoretical phase velocities for the vertical and horizontal components (e.g., Dal Moro and Ferigo, 2011), the different mode energy can be explained by the different amplitude between horizontal and vertical particle motions of Rayleigh waves. Boaga et al. (2013) also demonstrate that the use of the horizontal component of Rayleigh waves is effective in preventing mode misidentification. These results indicate that the horizontal component of Rayleigh waves plays an important role in multimode analysis of surface waves.

In this paper, we investigate the characteristics of multimode Rayleigh waves in the vertical and horizontal components to clarify the advantages of using the horizontal component of Rayleigh waves. We conduct a numerical simulation study to reveal the effects of source types and source depths on multimode Rayleigh waves in layered models with and without a low-velocity layer. We then confirm the findings of the simulation study by applying surface-wave analysis to $3 \mathrm{C}$ field data acquired in Alberta, Canada.

\section{NUMERICAL SIMULATIONS}

We first conduct a numerical simulation study to reveal the characteristics of multimode Rayleigh waves in multicomponent data. We use two kinds of layered models, model A and model B (Tables 1 and 2), in the simulation study. Anelastic attenuation is considered by assigning quality factors for P-waves $\left(Q_{\mathrm{P}}\right)$ and $\mathrm{S}$-waves $\left(Q_{\mathrm{S}}\right)$ to each layer. In model $\mathrm{A}, \mathrm{P}$ - and $\mathrm{S}$-wave velocities gradually increase with depth. It is well known that with the velocity increasing model (i.e., model A), the fundamental mode of Rayleigh waves is dominant at most frequencies in the vertical component data generated by a vertical source at the surface. In contrast, when a low-velocity layer is embedded among the stiffer layers as in model B, multimode surface waves are dominant in seismic data acquired in a near-surface seismic survey (e.g., Gucunski and Woods, 1992; Tokimatsu et al., 1992). Here, we use the discrete

Table 1. Parameters of model A.

\begin{tabular}{lccccccc}
\hline $\begin{array}{l}\text { Layer } \\
\text { no. }\end{array}$ & $\begin{array}{c}\text { Thickness } \\
(\mathrm{m})\end{array}$ & $\begin{array}{c}\text { P-wave } \\
\text { velocity } \\
(\mathrm{m} / \mathrm{s})\end{array}$ & $\begin{array}{c}\text { S-wave } \\
\text { velocity } \\
(\mathrm{m} / \mathrm{s})\end{array}$ & $Q_{\mathrm{P}}$ & $Q_{\mathrm{S}}$ & $\begin{array}{c}\text { Density } \\
\left(\mathrm{g} / \mathrm{cm}^{3}\right)\end{array}$ \\
\hline 1 & 30 & 1000 & 200 & 40 & 20 & 1.6 \\
2 & 50 & 1500 & 350 & 60 & 30 & 1.8 \\
3 & 100 & 2000 & 600 & 100 & 50 & 2.0 \\
4 & $\infty$ & 3000 & 1500 & 150 & 75 & 2.2 \\
\hline
\end{tabular}

wavenumber integral method (Bouchon and Aki, 1977) to calculate synthetic waveforms for the two models. In the simulations, 48 receivers set from 18 to $300 \mathrm{~m}$ from the source at a spacing of $6 \mathrm{~m}$ are assumed. The source function is an $8-\mathrm{Hz}$ Ricker wavelet, and the time duration is $8 \mathrm{~s}$.

To estimate Rayleigh-wave dispersion curves from the vertical and horizontal (radial) components of numerically synthesized waveforms, we perform a multichannel analysis of surface waves (MASW) (Park et al., 1999) using a phase shift method by Park et al. (1998). In the MASW method, seismic data in the time-offset $(t-x)$ domain are transformed into the frequency-offset $(f-x)$ domain by a Fourier transform. The dispersion image in the velocity-frequency $(c-f)$ domain is then obtained by taking absolute values of an integration of seismic data in the $f$-x domain over offset $x$ with a phase shift. In this integration, seismic data in the $f-x$ domain are normalized by their absolute values.

\section{Model A}

\section{Comparison of source types}

We investigate the effects of source types on multimode Rayleigh waves in model A by using a vertical force and an explosive source. The vertical force simulates a sledge hammer, a vibrator, or an impactor usually used in a near-surface seismic survey. On the other hand, the explosive source simulates a dynamite source used in a large-scale seismic reflection survey.

Dispersion images are estimated by MASW from the vertical and horizontal components of simulated data for the vertical force at $1 \mathrm{~m}$ below the surface (Figure 1). Amplitudes of the dispersion images are normalized by the maximum values by frequency. The theoretical dispersion curves shown in Figure 1 are computed by the compound matrix method (Saito, 1988; Saito and Kabasawa, 1993; Ikeda and Matsuoka, 2013). From the dispersion images, phase velocities with the absolute and relative energy maxima are picked. The relative maxima are defined as the maxima with energy more than $50 \%$ of the absolute maxima for the frequency. The maximum detectable wavelengths depend mainly on the distance between the minimum and maximum offsets (spread length) (e.g., Boiero and Socco, 2011). In this simulation study, we pick phase velocities with wavelengths up to twice as long as the spread length because they mostly agree with theoretical phase velocities.

The estimated dispersion curves (Figure 1) are mostly consistent with the theoretical dispersion curve of the fundamental mode. The energy of the dispersion curve estimated from the horizontal component is shifted to the first higher mode at $\sim 2 \mathrm{~Hz}$. To explain the observed mode energy theoretically, we compute theoretical

Table 2. Parameters of model B.

\begin{tabular}{lcccccc}
\hline $\begin{array}{l}\text { Layer } \\
\text { no. }\end{array}$ & $\begin{array}{c}\text { Thickness } \\
(\mathrm{m})\end{array}$ & $\begin{array}{c}\text { P-wave } \\
\text { velocity } \\
(\mathrm{m} / \mathrm{s})\end{array}$ & $\begin{array}{c}\text { S-wave } \\
\text { velocity } \\
(\mathrm{m} / \mathrm{s})\end{array}$ & $Q_{\mathrm{P}}$ & $Q_{\mathrm{S}}$ & $\begin{array}{c}\text { Density } \\
\left(\mathrm{g} / \mathrm{cm}^{3}\right)\end{array}$ \\
\hline 1 & 30 & 1500 & 350 & 60 & 30 & 1.8 \\
2 & 20 & 1000 & 200 & 40 & 20 & 1.6 \\
3 & 75 & 2000 & 600 & 100 & 50 & 2.0 \\
4 & $\infty$ & 3000 & 1500 & 150 & 75 & 2.2 \\
\hline
\end{tabular}


Rayleigh-wave ellipticities (amplitude ratio between horizontal and vertical particle motions) and the vertical and horizontal amplitude responses for a vertical force at the surface (Figure 2). The amplitude response of the fundamental mode is dominant in the vertical component (Figure 2b). In the horizontal component, however, the amplitude response of the first higher mode is dominant at $\sim 2 \mathrm{~Hz}$ because the trough of the Rayleigh-wave ellipticity for the fundamental mode at $\sim 2 \mathrm{~Hz}$ causes the amplitude of the fundamental mode in the horizontal component to be low (Figure $2 \mathrm{a}$ and $2 \mathrm{c}$ ). This result is consistent with the mode transition in the dispersion curve at $\sim 2 \mathrm{~Hz}$ in the horizontal component data (Figure 1b).

When the explosive source is buried at 1-m depth (Figure 3), the dispersion image for the vertical component data is similar to that for the vertical force. In the horizontal component, the energy of the first higher mode is dominant over a wider frequency range than the case of the vertical force, and the second higher mode is also dominant at $\sim 5 \mathrm{~Hz}$ (Figure 3b). Picking relative maxima is helpful in
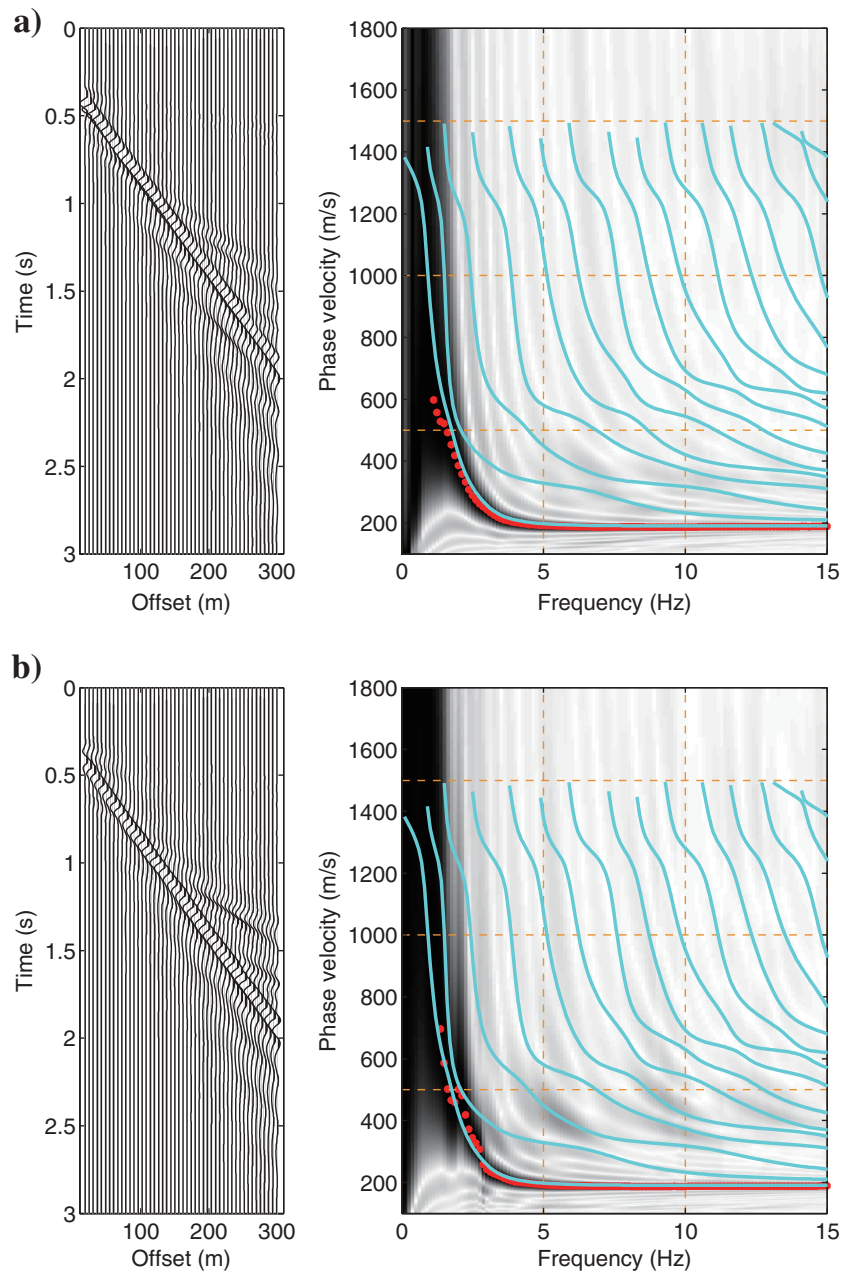

Figure 1. The 30-Hz low-pass-filtered synthesized waveforms and dispersion images for (a) vertical and (b) horizontal component data when the vertical force is located at 1-m depth in model A. The red circles are phase velocities with absolute maxima for each frequency, and the cyan lines are theoretical dispersion curves. recognizing additional higher modes (Socco and Strobbia, 2004) in the horizontal component data.

\section{Comparison of source depths}

Explosive sources (e.g., dynamite) are usually buried under the ground so that the explosive energy is effectively transferred to the geologic formation. The buried sources might improve the resolution at depth (Duputel et al., 2010). In our simulations, we therefore deploy explosive sources at depths of 8 and $15 \mathrm{~m}$ to evaluate the effect of source depth. In the vertical component data for the explosive source at 8 and $15 \mathrm{~m}$ depth (Figures $4 \mathrm{a}$ and 5a), the body waves have larger relative amplitudes at the greater source depth. Therefore, we mute the body waves in the vertical component data (red lines in Figures $4 \mathrm{a}$ and 5a) before applying MASW. When the explosive source is located at 8-m depth (Figure 4), several higher mode signals can be clearly identified in the horizontal component data at high frequencies. In contrast, the fundamental mode is mostly dominant in the vertical component data, although several modes can be estimated by picking relative maxima. Higher-mode
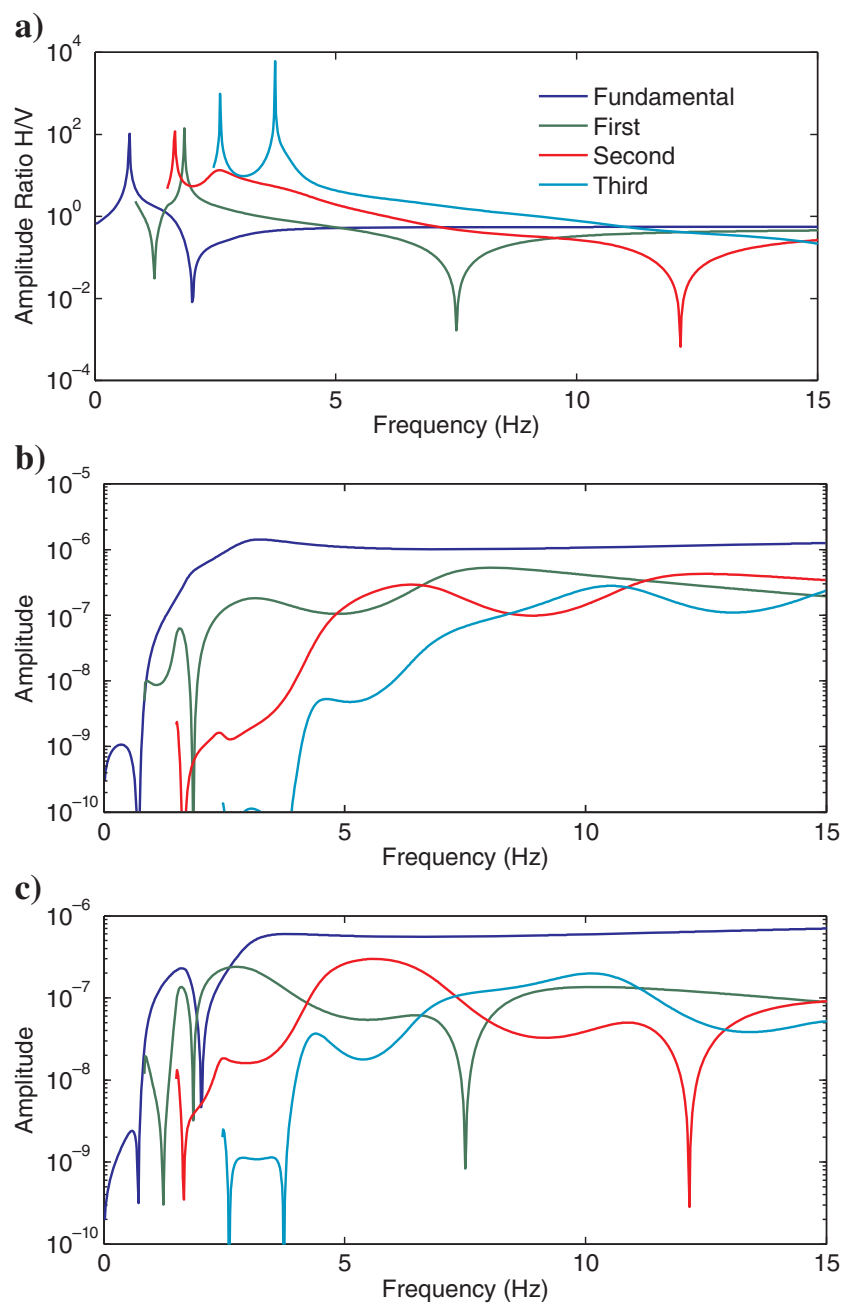

Figure 2. (a) Theoretical Rayleigh-wave ellipticities and (b) vertical and (c) horizontal amplitude responses up to the third higher mode for model A. 
signals are dominant in the horizontal and vertical components for the explosive source at 15-m depth (Figure 5). However, the modes and their frequency ranges imaged in the dispersion image from the horizontal component data (Figure 5b) differ from those imaged from the vertical component data (Figure 5a). As a result, the picked phase velocities for each mode cover different frequency ranges depending on the components (vertical or horizontal).

These results demonstrate that clearer mode information can be extracted when the horizontal component data are used along with the vertical component data. Note that, although muting body waves improves the extraction of surface-wave dispersion curves at higher frequencies, it generates artificial phase velocities at low frequencies ( $<2 \mathrm{~Hz}$; Figures 4a and 5a) (Ivanov et al., 2005). However, they can be removed by comparing dispersion images with or without muting. Therefore, we neglect them in the following analysis. We also note that several observed phase velocities exceed the maximum $\mathrm{S}$-wave velocity of the simulated model $(1500 \mathrm{~m} / \mathrm{s})$; these might be explained by guided P-waves (Boiero et al., 2013). a)

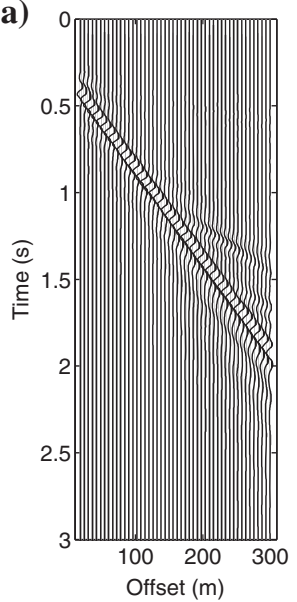

b)

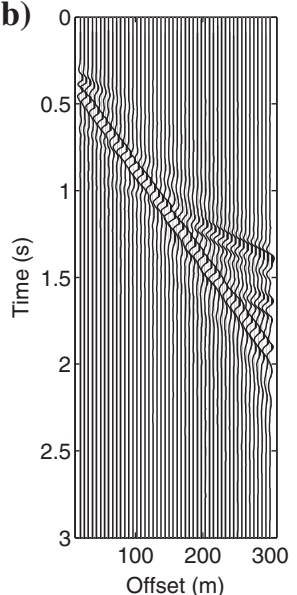

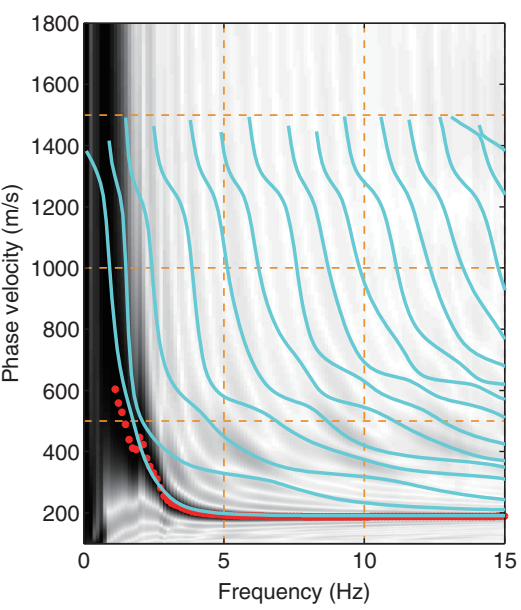

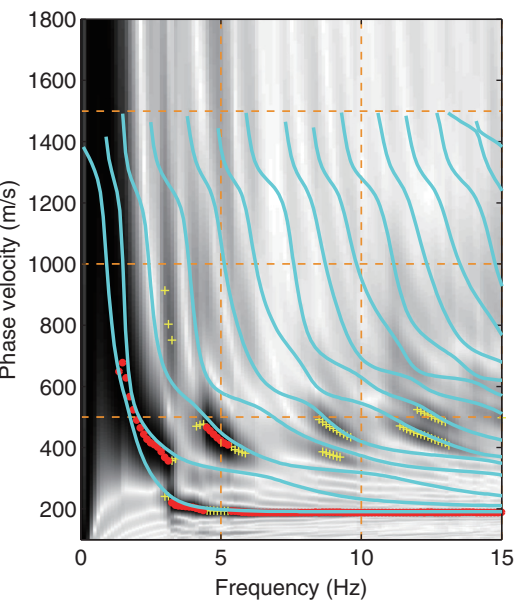

Figure 3. The 30-Hz low-pass-filtered synthesized waveforms and dispersion images for (a) vertical and (b) horizontal component data when the explosive source is located at 1-m depth in model A. The red circles and yellow crosses are phase velocities with absolute maxima and relative maxima for each frequency, respectively. The cyan lines are theoretical dispersion curves.

\section{Model B}

\section{Comparison of source types}

A smooth mode transition can be observed in the estimated dispersion curves of both components when the vertical force is located at 1-m depth for model B (Figure 6). These features are typical when a low-velocity layer is embedded among the layers (e.g., Gucunski and Woods, 1992; Tokimatsu et al., 1992; Tsuji et al., 2012). However, transit frequencies of the dominant modes in the horizontal component data differ slightly from those in the vertical component data. We also compute the theoretical Rayleigh-wave ellipticities and the vertical and horizontal amplitude responses for a vertical force at the surface for model B (Figure 7). The mode transition of the vertical component data is consistent with that of the vertical amplitude response (Figure 7b). At $\sim 2 \mathrm{~Hz}$, the horizontal amplitude response of the fundamental mode suddenly decreases and the first higher mode becomes dominant because of the trough in the Rayleigh-wave ellipticity of the fun-
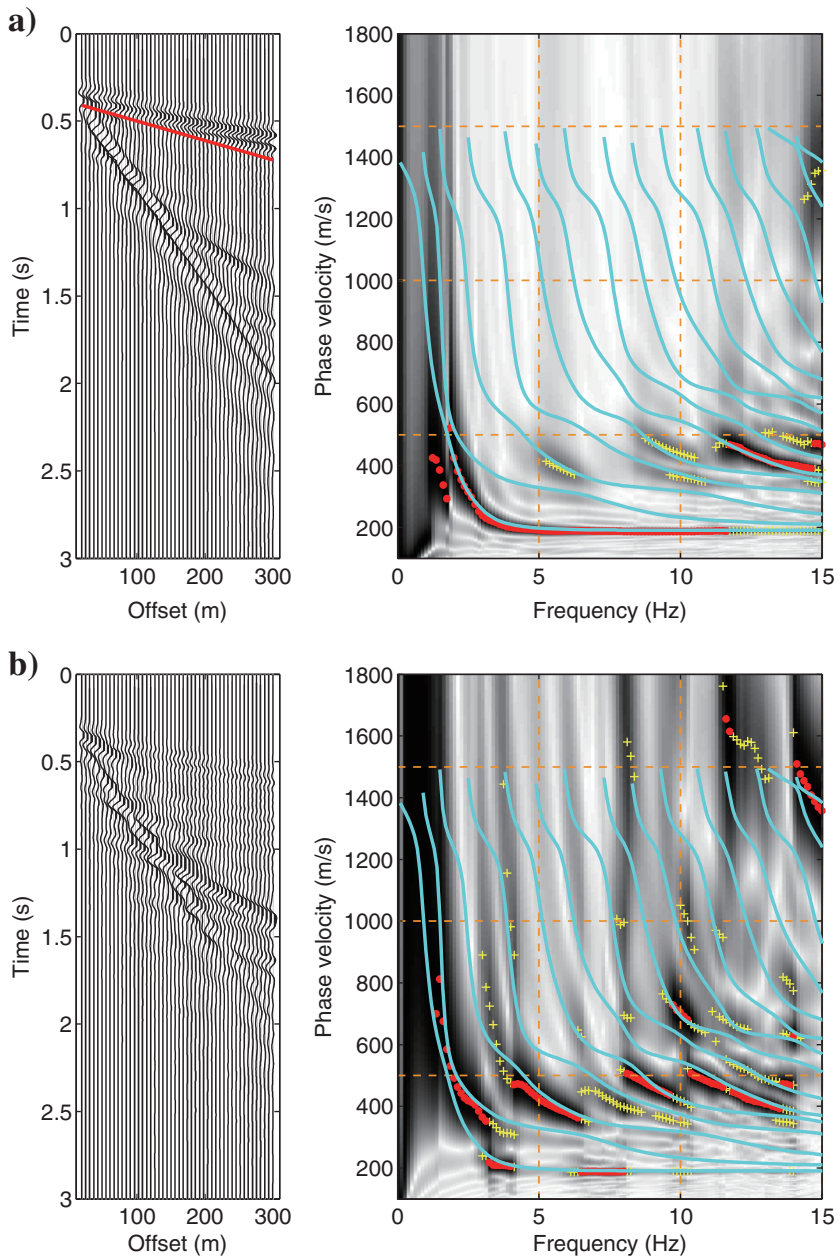

Figure 4. The 30-Hz low-pass-filtered synthesized waveforms and dispersion images for (a) vertical and (b) horizontal component data when the explosive source is located at an 8-m depth in model A. The body waves of the vertical component data are muted (red line). The red circles and yellow crosses are phase velocities with absolute maxima and relative maxima for each frequency, respectively. The cyan lines are theoretical dispersion curves. 
damental mode at $\sim 2 \mathrm{~Hz}$ (Figure $7 \mathrm{a}$ and $7 \mathrm{~d}$ ). Therefore, the fundamental mode vanishes at $\sim 2 \mathrm{~Hz}$ in the horizontal component data (Figure 6b). The relative maxima at $\sim 5 \mathrm{~Hz}$ (yellow crosses in Figure $6 b$ ) correspond to the horizontal amplitude response of the second higher mode (Figure 7c). The mode transition among the fundamental mode, first higher mode, and third higher mode at $\sim 7.5 \mathrm{~Hz}$ (Figure 6b) also agrees with the horizontal amplitude response (Figure 7e).

When the explosive is set at 1-m depth (Figure 8), the absolute maxima show similar mode transitions to those with the vertical force. By picking relative maxima in the horizontal component, however, a large number of modes can be identified.

\section{Comparison of source depths}

When the explosive source is located at 8-m depth (Figure 9), the absolute maxima for the vertical component data are similar to that estimated for the explosive source at 1-m depth (Figure 8a).
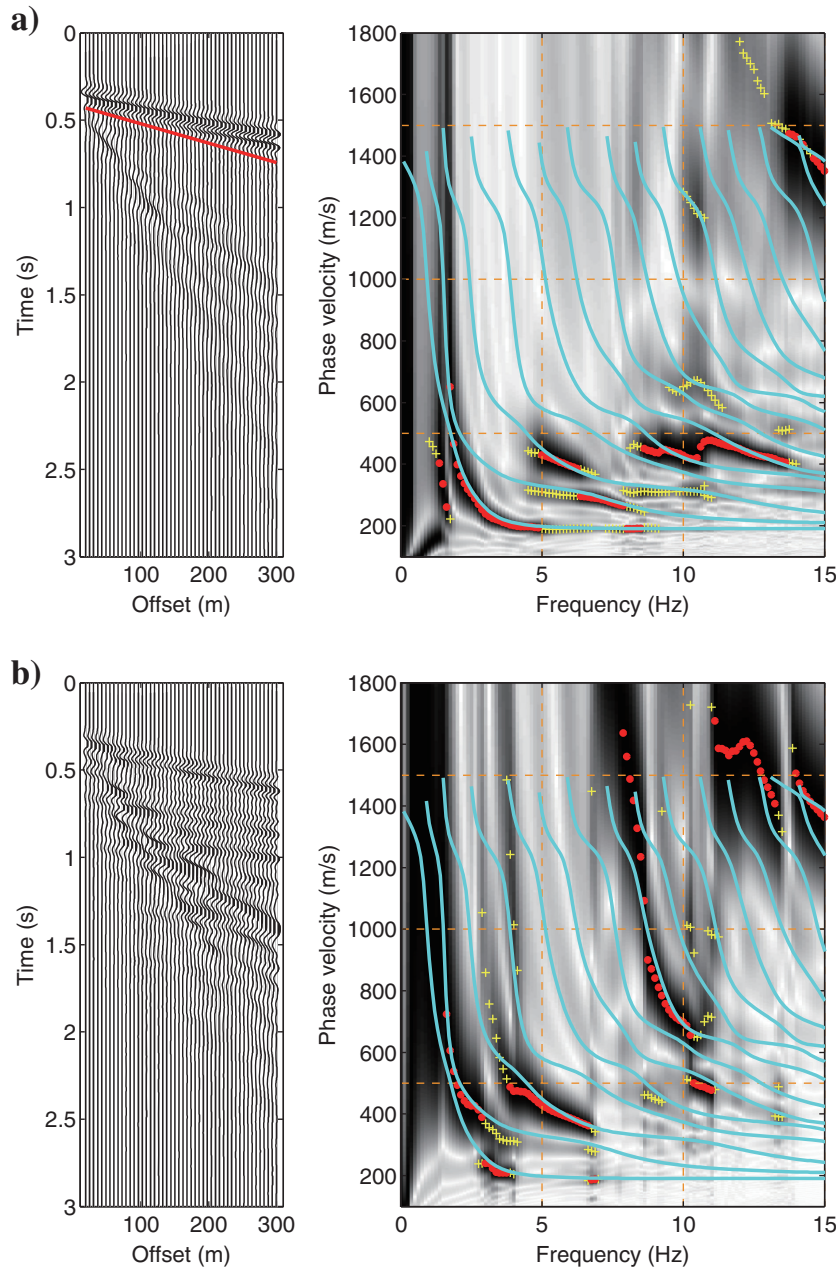

Figure 5. The 30-Hz low-pass-filtered synthesized waveforms and dispersion images for (a) vertical and (b) horizontal component data when the explosive source is located at $15-\mathrm{m}$ depth in model A. The body waves of the vertical component data are muted (red line). The red circles and yellow crosses are phase velocities with absolute maxima and relative maxima for each frequency, respectively. The cyan lines are theoretical dispersion curves.
However, the picked relative maxima provide additional mode information. In contrast, the dominant modes in the horizontal component data are shifted to higher-order modes with higher phase velocities (Figure 9b). When the explosive source is located at a 15-m depth (Figure 10), we mute the strong body waves in the vertical component data (red line in Figure 10a). The modes extracted from the vertical component data are shifted to higherorder modes, but it is apparent that in the horizontal component data, the higher-order modes with higher phase velocities have larger relative amplitudes, compared with the vertical component data. Thus, additional mode information can also be extracted from the horizontal component data for model B.

\section{Sensitivity analysis}

The numerical simulation study demonstrates that several multimode phase velocities can be extracted when the explosive source is buried. To assess the importance of multimode Rayleigh waves
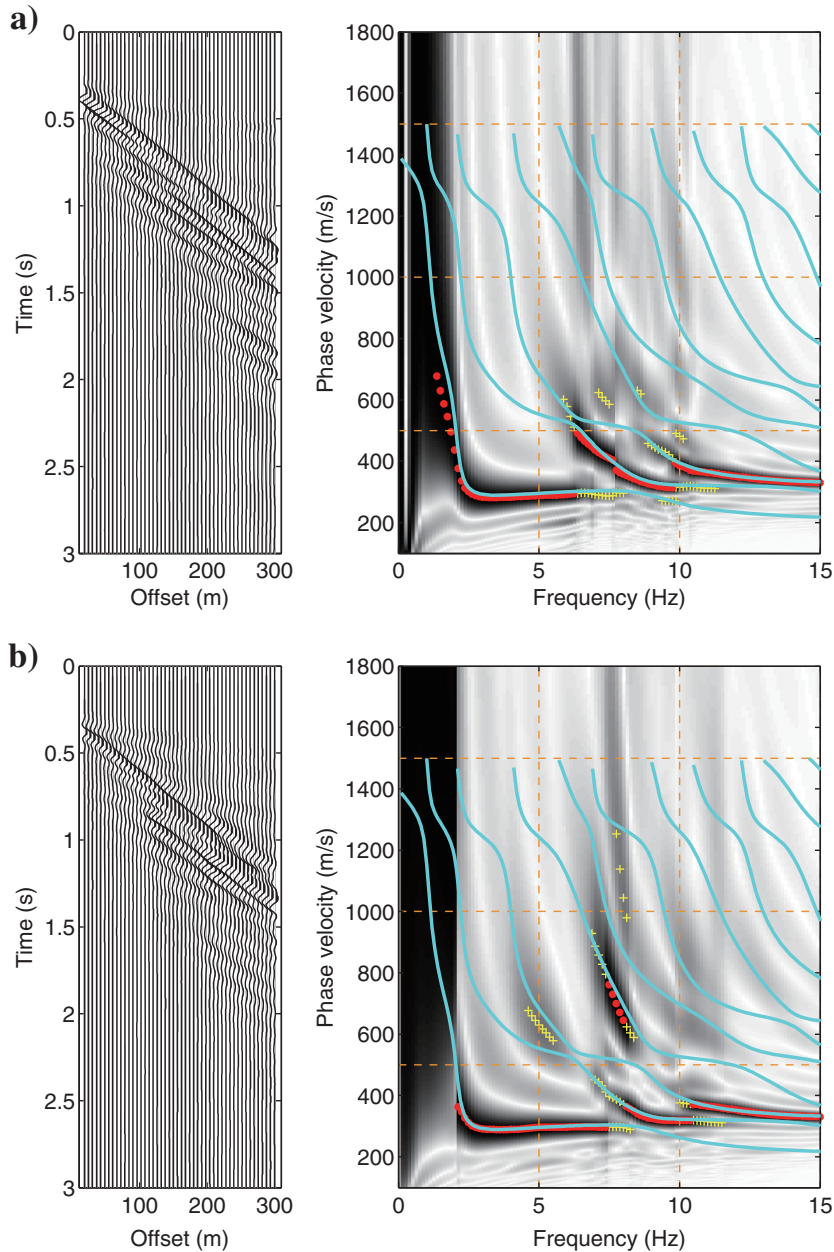

Figure 6. The 30-Hz low-pass-filtered synthesized waveforms and dispersion images for (a) vertical and (b) horizontal component data when the vertical force is located at 1-m depth in model B. The red circles and yellow crosses are phase velocities with absolute maxima and relative maxima for each frequency, respectively. The cyan lines are theoretical dispersion curves. 
extracted from the vertical and horizontal component data for the explosive source at a 15-m depth (Figures 5 and 10), we investigate the sensitivity of multimode dispersion curves to $\mathrm{S}$-wave velocity changes. We compare observed phase velocities with theoretical dispersion curves obtained by changing the S-wave velocities within a range of $\pm 10 \%$ in each layer. In the results of sensitivity analyses (Figures 11 and 12), black lines denote theoretical
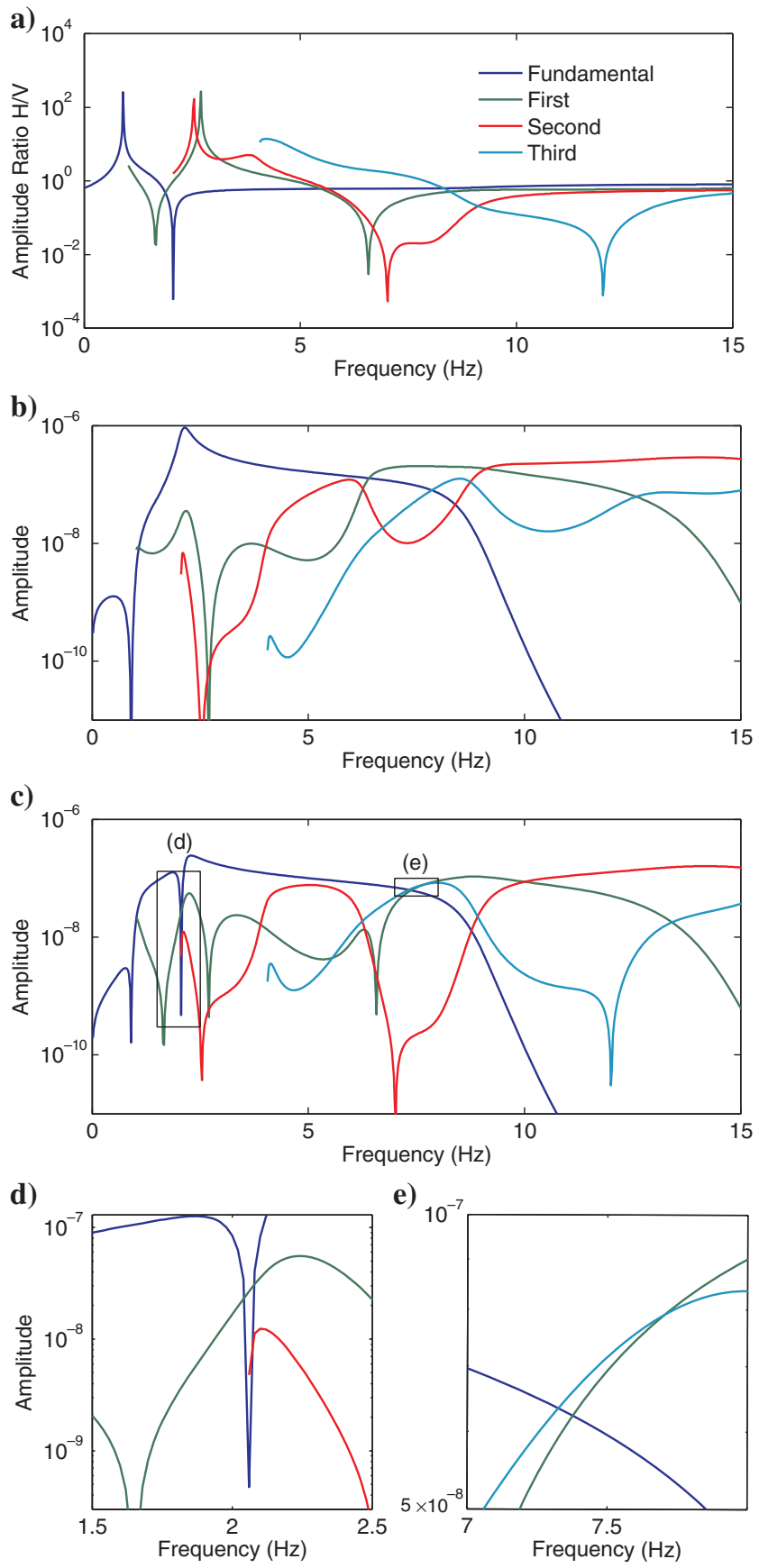

e)

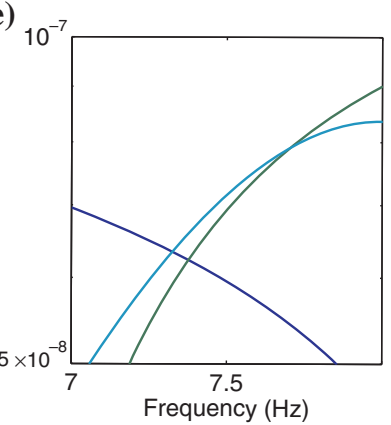

Figure 7. (a) Theoretical Rayleigh-wave ellipticities and (b) vertical and (c) horizontal amplitude responses up to the third higher mode for model B. (d and e) Enlarged view of the horizontal amplitude responses. dispersion curves for each model. Theoretical dispersion curves with the S-wave velocity changes for each layer are described in colored lines corresponding to the $\mathrm{S}$-wave velocity changes. Purple and red marks are phase velocities with absolute and relative maxima estimated from the vertical and horizontal component data, respectively.

Compared with the fundamental mode, higher modes show a greater sensitivity to the $\mathrm{S}$-wave velocity changes in the same layers with the increase of frequencies. In model A (Figure 11), the dispersion curves of the first and seventh higher modes extracted from the horizontal component data show sensitivity to S-wave velocity changes in the third layer (red marks in Figure 11c). In contrast, the dispersion curves estimated from the vertical component data show a small sensitivity to velocity changes in the third layer (purple marks in Figure 11c). In model B (Figure 12), phase velocities extracted from the vertical component data are mostly sensitive to S-wave velocity changes in the first and second layers (purple marks in Figure 12). However, higher modes extracted from
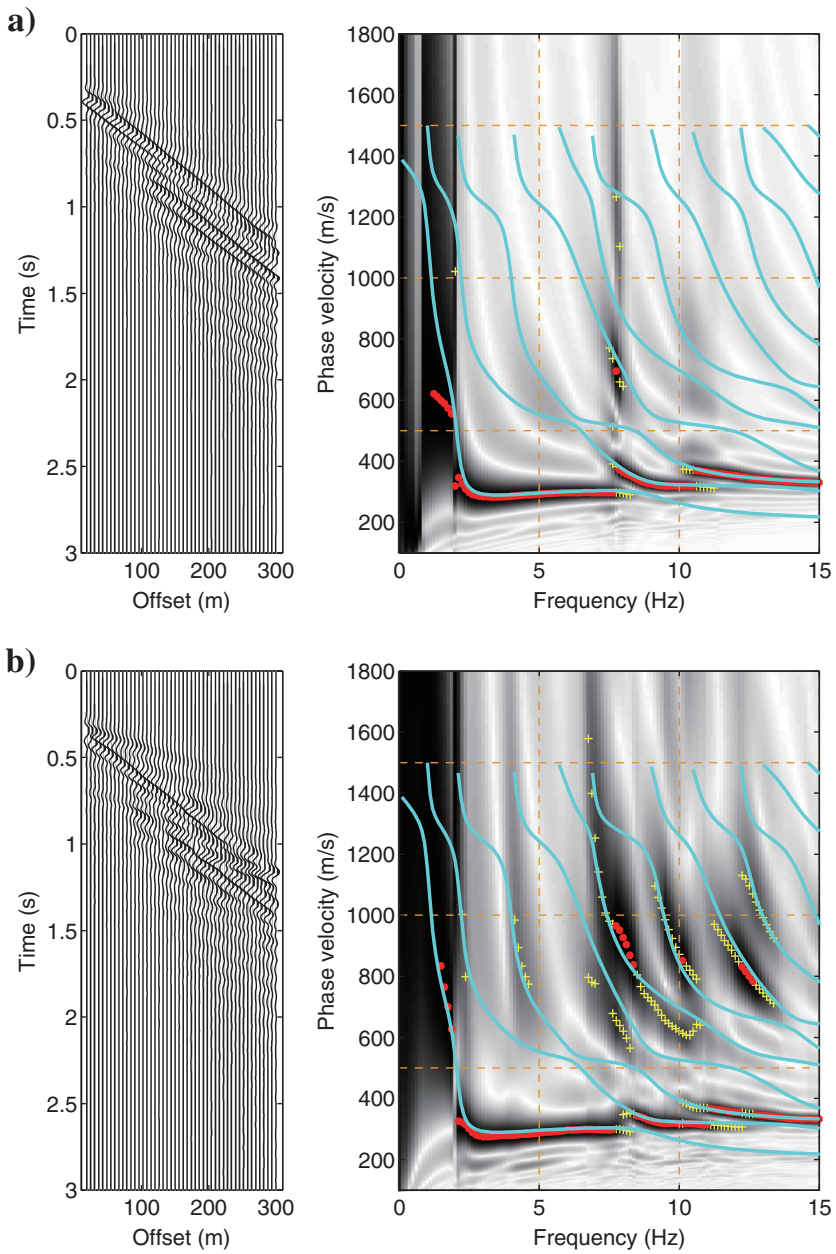

Figure 8 . The $30-\mathrm{Hz}$ low-pass-filtered synthesized waveforms and dispersion images for (a) vertical and (b) horizontal component data when the explosive source is located at 1-m depth in model B. The red circles and yellow crosses are phase velocities with absolute maxima and relative maxima for each frequency, respectively. The cyan lines are theoretical dispersion curves. 
the horizontal component data show some amount of sensitivity to velocity changes in the third layer as in the case of the first and second layers (red marks in Figure 12).

\section{FIELD EXAMPLE}

We apply a surface-wave analysis to field data and compare the characteristics of the multicomponent Rayleigh waves with those from the simulation study.

\section{Data acquisition}

The field data were originally acquired for monitoring variation of P-wave velocity associated with steam-assisted gravity drainage in Alberta, Canada, by Japan Canada Oil Sands Limited in 2002 and 2006 (Kato et. al., 2008; Nakayama et al., 2008). We apply a surface-wave analysis to a shot gather of the field data acquired in 2006 (Figure 13). The receivers were 3C digital sensors incorporating microelectromechanical systems technology (Sercel DSU3). a)

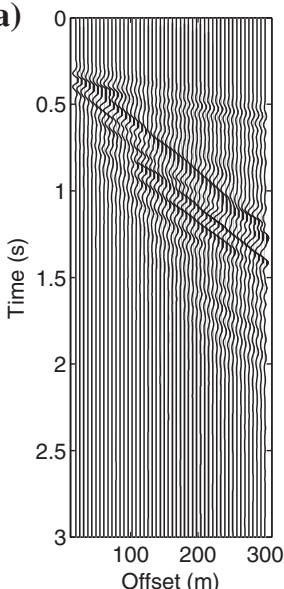

b)

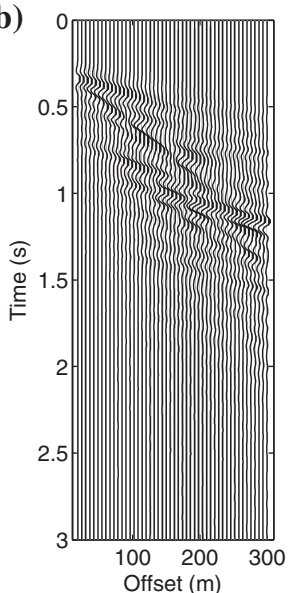

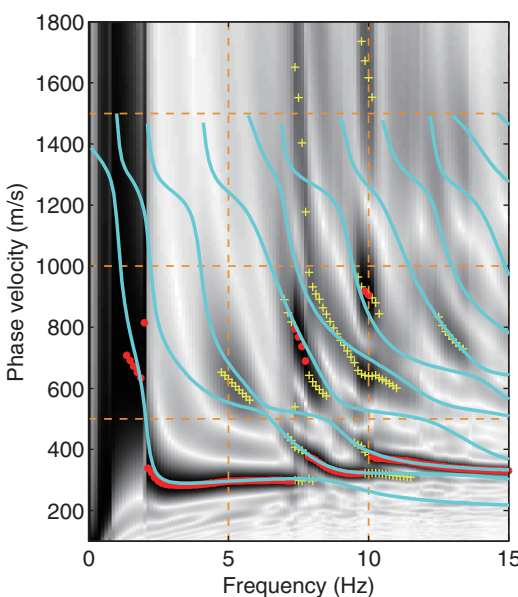

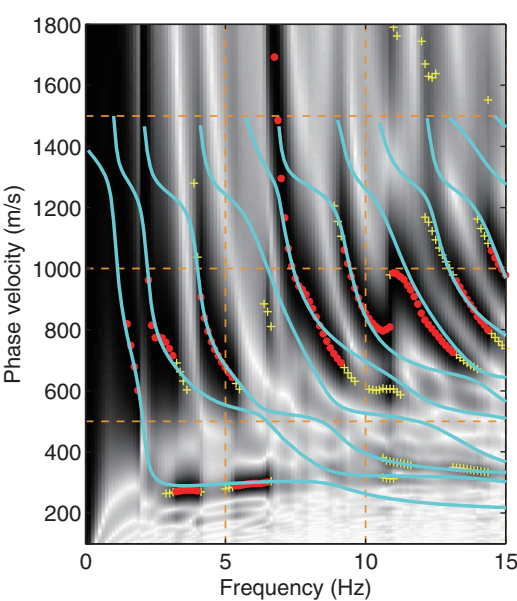

Figure 9. The 30-Hz low-pass-filtered synthesized waveforms and dispersion images for (a) vertical and (b) horizontal component data when the explosive source is located at an 8-m depth in model B. The red circles and yellow crosses are phase velocities with absolute maxima and relative maxima for each frequency, respectively. The cyan lines are theoretical dispersion curves.
DSU3 offers a broadband linear phase and amplitude response (Mougenot, 2006), which covers the response at low frequencies used in this analysis. The sampling interval was $1 \mathrm{~ms}$, and the record length was $3 \mathrm{~s}$. The dynamite source $(0.125 \mathrm{~kg})$ was positioned at a $15-\mathrm{m}$ depth. This source configuration is similar to that for models $A$ and B with an explosive source at a 15-m depth (Figures 5 and 10) in the simulation study. Although the maximum offset between the source and receivers was originally $815 \mathrm{~m}$, we use only the receivers within $300 \mathrm{~m}$ from the source as in Figure 13 because of the 3-s record length. In this field example, the choice of the minimum offset (originally $5.3 \mathrm{~m}$ ) is not sensitive in estimating phase velocities up to wavelengths close to the spread length. Therefore, we pick wavelengths up to the spread length by using all seismic traces within $300 \mathrm{~m}$ from the source.

\section{Dispersion-curve estimations}

This oil field can be assumed as a horizontally layered structure (Takahashi, 2010), and therefore, we estimate one dispersion image
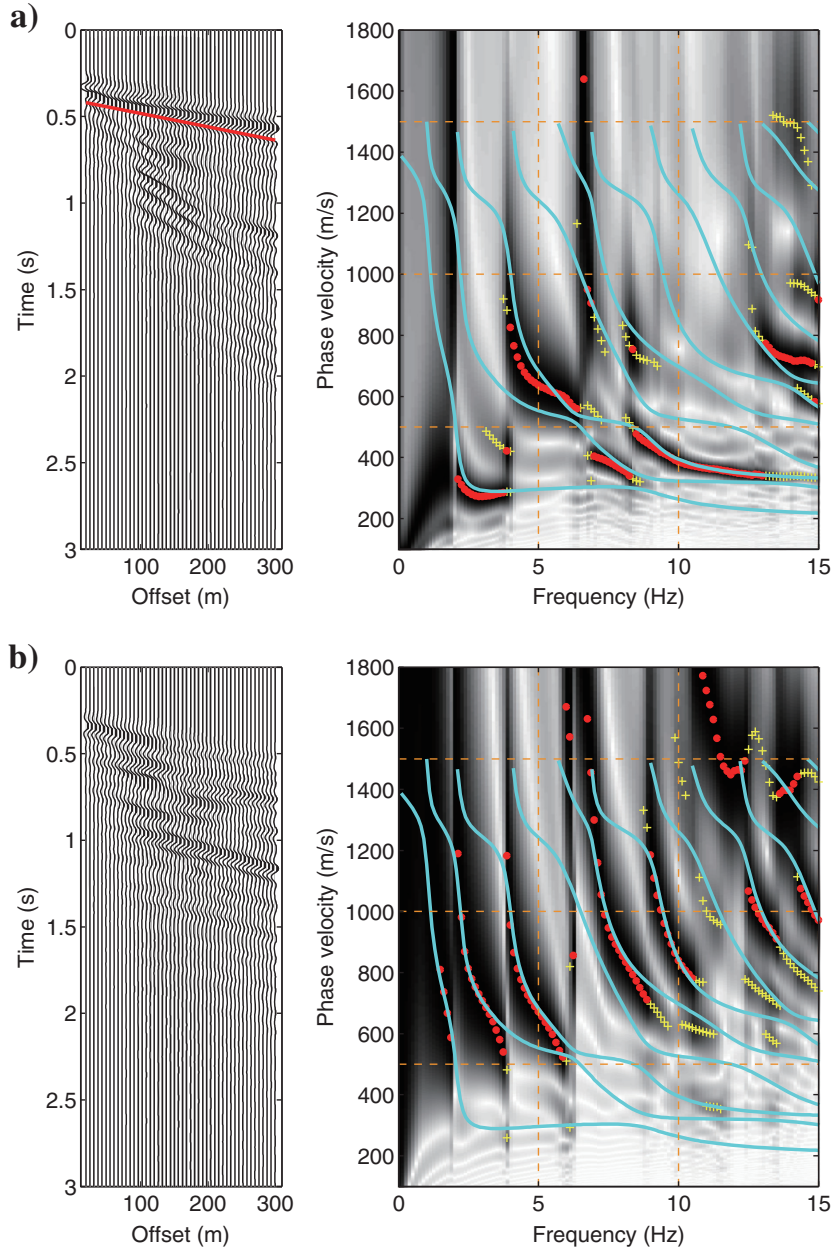

Figure 10. The $30-\mathrm{Hz}$ low-pass-filtered synthesized waveforms and dispersion images for (a) vertical and (b) horizontal component data when the explosive source is located at a $15-\mathrm{m}$ depth in model B. Body waves of the vertical component data are muted (red line). The red circles and yellow crosses are phase velocities with absolute maxima and relative maxima for each frequency, respectively. The cyan lines are theoretical dispersion curves. 


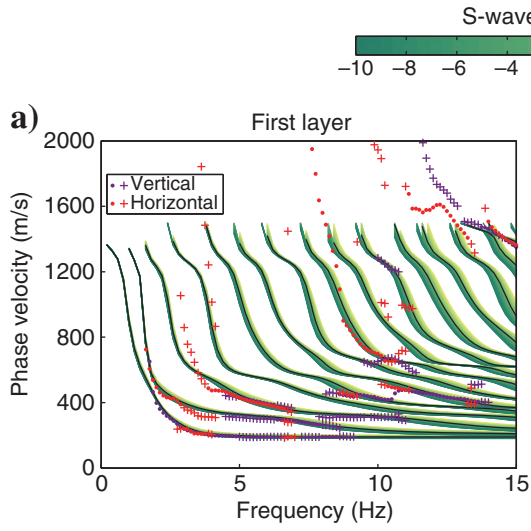

-wave velocity change (\%)
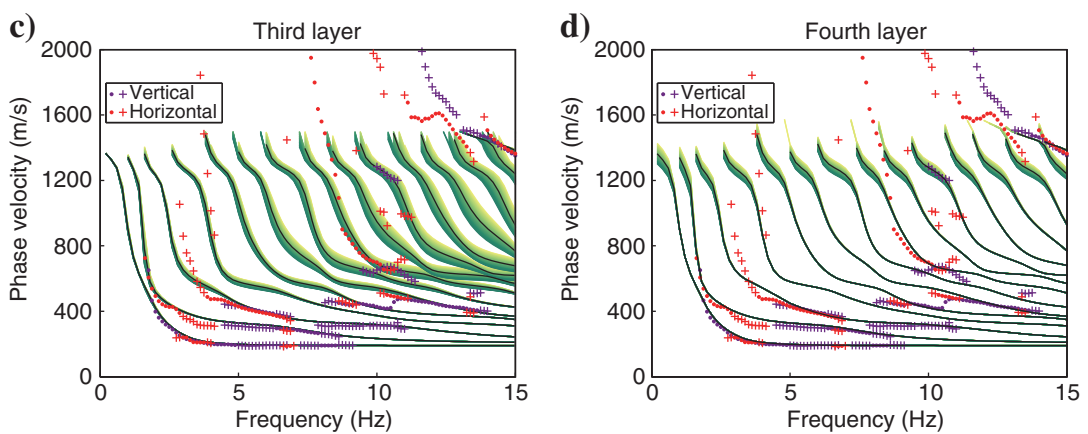

Figure 11. Sensitivity analysis results for the (a) first, (b) second, (c) third, and (d) fourth layers of model A. S-wave velocities of each layer are changed within a range of $\pm 10 \%$ for the simulated model. Circles and crosses are the observed phase velocities with absolute maxima and relative maxima, respectively.
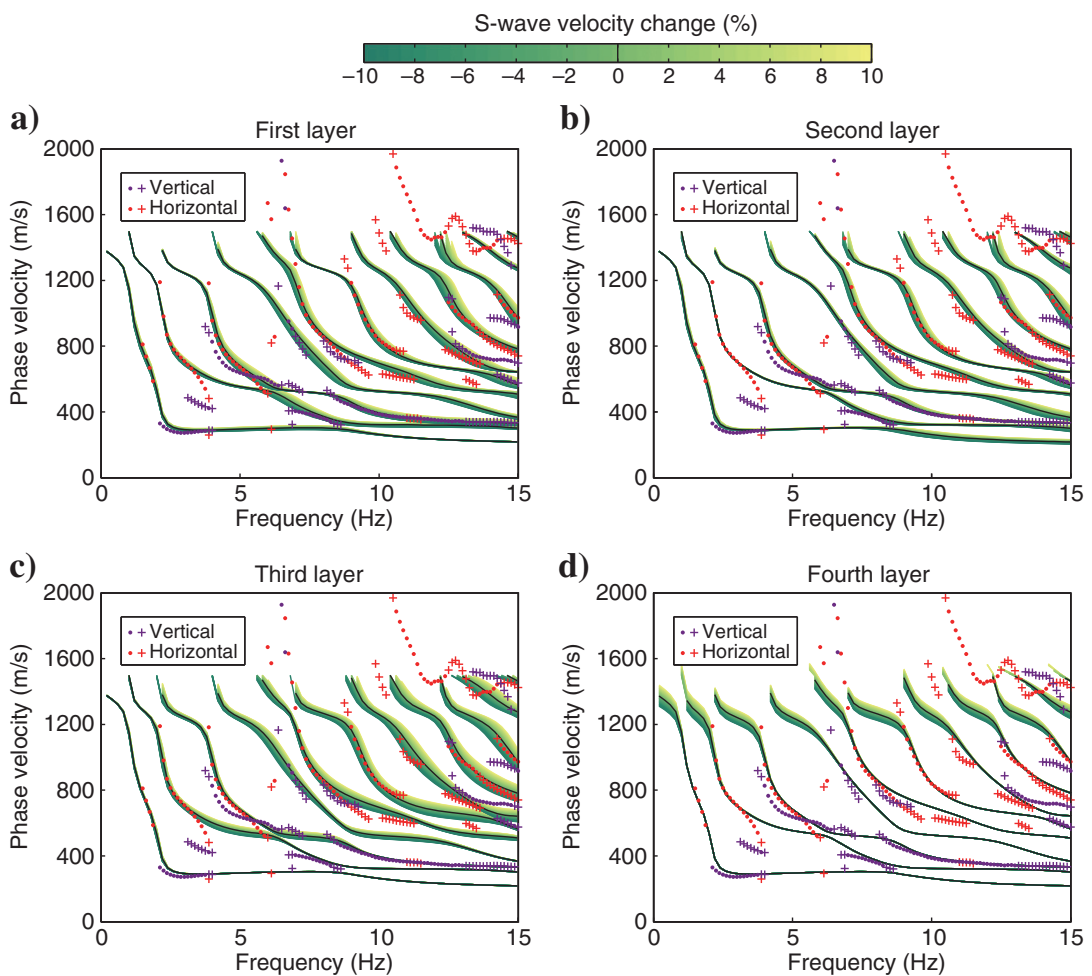

Figure 12. Sensitivity analysis results for the (a) first, (b) second, (c) third, and (d) fourth layers of model B. S-wave velocities of each layer are changed within a range of $\pm 10 \%$ for the simulated model. Circles and crosses are the observed phase velocities with absolute maxima and relative maxima, respectively. from each component of the seismic data acquired by the two-dimensionally distributed receivers (Figures 13 and 14). The P-SV waves in radial components and $\mathrm{SH}$-waves in transverse components are calculated from the two horizontal components of north-south and east-west directions by considering the geometries between the source and receivers. The observed shot gathers and dispersion images estimated by MASW for the vertical and horizontal components of the P-SV waves are shown in Figure 14. Love waves are not observed in the dispersion image of the horizontal component of the SH waves (not shown) because SH waves are not induced by an explosive source (Aki and Richards, 1980).

Continuous phase velocities related to several modes of Rayleigh waves can be observed in each component (e.g., A-F in Figure 14). When continuous phase velocities are picked from both component data (Figure 15), we see that higherorder modes can be observed in the horizontal component data at the same frequencies. The mode of the dispersion curve in the frequency range from 4 to $8 \mathrm{~Hz}$ in the vertical component data (B in Figure 15) should be identical to the mode in the frequency range from 2 to $6 \mathrm{~Hz}$ in the horizontal component data (D in Figure 15) because they are overlapped in the frequency range from 4 to $6 \mathrm{~Hz}$. The different energy distribution for different components would be explained by the different amplitude between the horizontal and vertical particle motions of Rayleigh waves as demonstrated in Figures 2 and 7. Thus, additional mode information can be obtained by using the horizontal component data of the P-SV waves. These characteristics of the vertical and horizontal component data of multimode Rayleigh waves agree with the numerical simulation study performed with the same source configuration (i.e., an explosive source at 15-m depth; Figures 5 and 10).

\section{DISCUSSION}

The results of the simulation study and field example indicate that the analysis of the horizontal component of the Rayleigh waves allows us to use additional higher-order mode information when the explosive source is buried. Although the use of a buried explosive source and $3 \mathrm{C}$ receivers is not common in near-surface seismic surveys, it is possible to extract multimode Rayleigh waves from multicomponent data originally acquired for large-scale seismic reflection as the oil field seismic survey shown in the previous section. Estimation of shallow velocity models by surface-wave analysis can improve static corrections in seismic reflection surveys (Strobbia et al., 2010; R. Askari, personal communication, 2013; Boiero et al., 2013). 


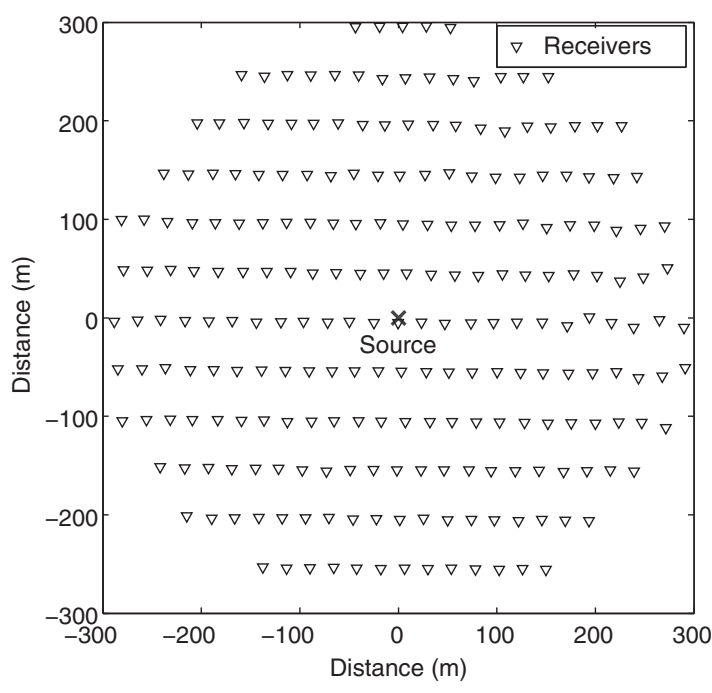

Figure 13. Positions of the source and the receivers used in the surface-wave analysis.
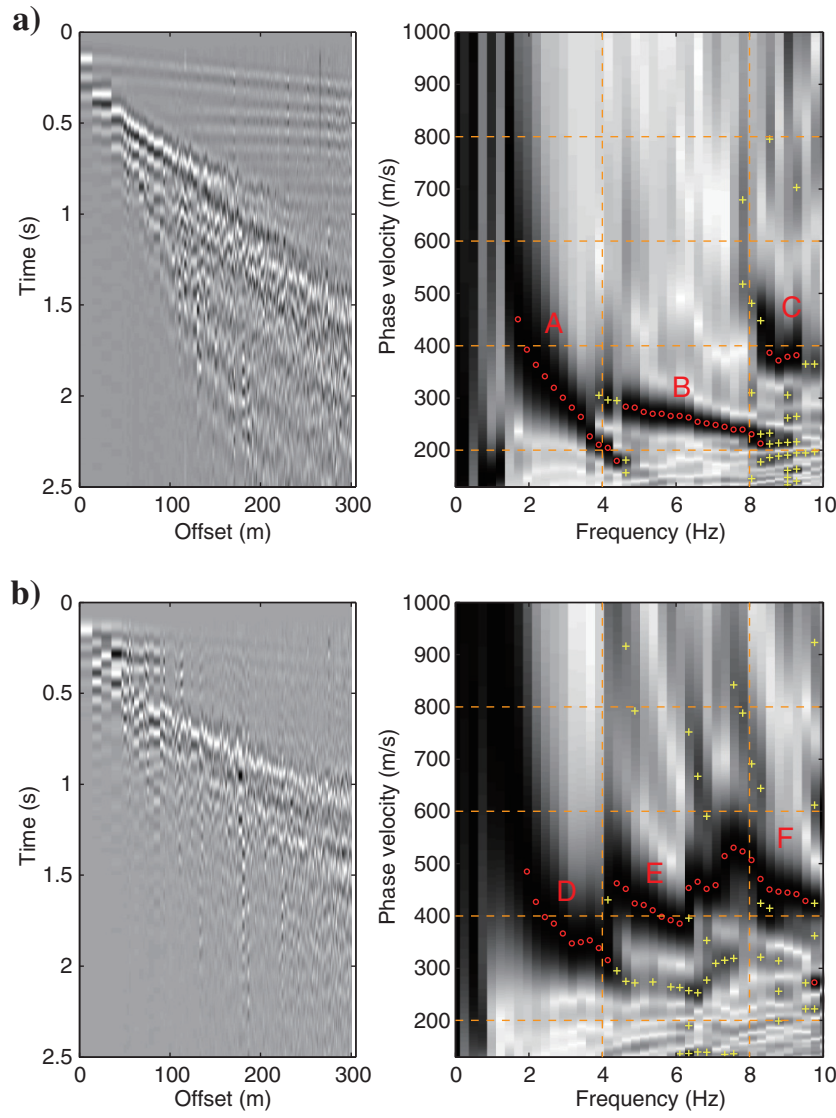

Figure 14. The 10-Hz low-pass-filtered seismic data and dispersion images for (a) vertical and (b) horizontal component data. Red circles and yellow crosses are phase velocities with absolute maxima and relative maxima for each frequency, respectively. The continuous maxima around $\mathrm{A}-\mathrm{F}$ are considered to be related to multimode Rayleigh waves.

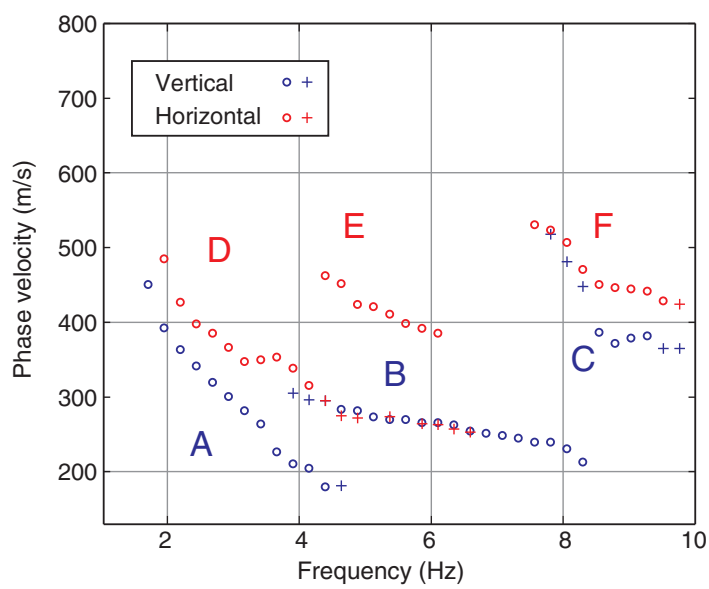

Figure 15. Dispersion curves estimated from the vertical and horizontal component data (A-F). Circles and crosses are picked phase velocities with absolute and relative maxima around $\mathrm{A}-\mathrm{F}$ in Figure 14, respectively.

As demonstrated in the simulation study, the different mode energy distributions of Rayleigh waves can be observed in dispersion images depending on source types and source depths. Dispersion images also depend on the minimum offset and spread length even if velocity models are the same (Ivanov et al., 2008; Ivanov et al., 2011). On the other hand, different models can have identical dispersion images within a given frequency range (Ivanov et al., 2013). Dispersion images from the horizontal component data can complement those from the vertical component data and, therefore, help resolve dispersion imaging deficiencies.

The results of this study also demonstrate that the additional use of multimode Rayleigh waves extracted from the horizontal components has the potential for improving S-wave velocity estimations. Many authors have demonstrated that the use of multimode surface waves in inversions can improve S-wave velocity estimations (e.g., Xia et al., 2003; Luo et al., 2007). However, we need to carefully perform inversion analysis, in particular, when several higher-order modes are used in inversion. We might fail to identify the correct mode number even if the horizontal component data are used (e.g., the third and fourth modes in Figure 11 and $\mathrm{C}$ and $F$ in Figure 15). To prevent such mode misidentification, we should use novel inversion techniques without mode identification (e.g., Forbriger, 2003; O’Neill et al., 2003; Ryden and Park, 2006; Maraschini et al., 2010; Socco et al., 2011; Boiero et al., 2013). Multimode Rayleigh waves extracted from the vertical and horizontal components can thus be used to improve S-wave velocity estimations by using appropriate multimode inversion techniques.

\section{CONCLUSIONS}

To examine the utility of the horizontal components of Rayleigh waves, we investigate the characteristics of the horizontal and vertical components of multimode Rayleigh waves. We conduct numerical modeling and field data analyses rather than a theoretical study for both components of Rayleigh waves.

The numerical simulation study demonstrates that when the source is located near the surface, absolute maxima of dispersion images in the horizontal component data have a trend similar to those from the vertical component data except at low frequencies. 
Picking relative maxima is useful to obtain additional multimode phase velocities from the horizontal component data. On the other hand, higher-order modes are dominant, especially in the horizontal component data, when the source depth of an explosive source is increased. Sensitivity analyses of S-wave velocity changes reveal that multimode Rayleigh waves extracted from the horizontal component data compensate the sensitivity of the vertical component data. Therefore, the use of horizontal component data with the vertical component data has the potential for improving S-wave velocity estimation. The higher-order modes are also observed from the horizontal components of the field data acquired by $3 \mathrm{C}$ receivers with a buried dynamite source.

Simulations and field studies demonstrate that additional mode information of Rayleigh waves can be extracted from the horizontal component data, in particular, acquired by an explosive source at a greater depth. The dispersion images from the horizontal component data can be effectively used to complement those from the vertical component data. As a result, the additional use of the horizontal components of Rayleigh waves would contribute to improving S-wave velocity structure estimations.

Data acquisition using an explosive source and $3 \mathrm{C}$ receivers is not common in surface seismic surveys. However, there are many existing data sets from large-scale seismic reflection surveys that were acquired in this way. Therefore, our results also contribute to constructing accurate near-surface S-wave velocity models for static corrections in analyses of seismic reflection data.

\section{ACKNOWLEDGMENTS}

We appreciate the helpful discussions and comments from A. Takahashi at Japan Petroleum Exploration Company Limited (JAPEX). We thank M. Asten at Monash University for the useful comments on this manuscript. We also thank M. Cara, J. Ivanov, and an anonymous referee and associate editor L. V. Socco for constructive comments. T. Ikeda is grateful for support provided by a grant-in-aid for JSPS Fellows (246036). We are grateful for support of the SATREPS grant from JST/JICA. T. Tsuji and T. Ikeda gratefully acknowledge the support of the International Institute for Carbon Neutral Energy Research (WPI-I2CNER), sponsored by the World Premier International Research Center Initiative, MEXT, Japan. The 3C-3D seismic data acquisition was conducted as part of a joint project between Japan Canada Oil Sands Limited (JACOS) and Japan Oil, Gas and Metals National Corporation (JOGMEC). The authors thank JOGMEC, JACOS, and JAPEX for the permission to use the $3 \mathrm{C}$ seismic data set and publish these data.

\section{REFERENCES}

Aki, K., and P. Richards, 1980, Quantitative seismology: Theory and methods: W.H. Freeman \& Co.

Boaga, J., G. Cassiani, C. L. Strobbia, and G. Vignoli, 2013, Mode misidentification in Rayleigh waves: Ellipticity as a cause and a cure: Geophysics, 78, no. 4, EN17-EN28, doi: 10.1190/geo2012-0194.1.

Boiero, D., and L. V. Socco, 2011, The meaning of surface wave dispersion curves in weakly laterally varying structures: Near Surface Geophysics, 9, 561-570, doi: 10.3997/1873-0604.2011042.

Boiero, D., E. Wiarda, and P. Vermeer, 2013, Surface- and guided-wave inversion for near-surface modeling in land and shallow marine seismic data: The Leading Edge, 32, 638-646, doi: 10.1190/tle32060638.1.

Bouchon, M., and K. Aki, 1977, Discrete wavenumber representation of seismic wave fields: Bulletin of the Seismological Society of America, 67, 259-277.
Dal Moro, G., and F. Ferigo, 2011, Joint analysis of Rayleigh- and Lovewave dispersion: Issues, criteria and improvements: Journal of Applied Geophysics, 75, 573-589, doi: 10.1016/j.jappgeo.2011.09.008.

Duputel, Z., M. Cara, L. Rivera, and G. Herquel, 2010, Improving the analysis and inversion of multimode Rayleigh-wave dispersion by using groupdelay time information observed on arrays of high-frequency sensors: Geophysics, 75, no. 2, R13-R20, doi: 10.1190/1.3341172.

Forbriger, T., 2003, Inversion of shallow-seismic wavefields: II. Inferring subsurface properties from wavefield transforms: Geophysical Journal International, 153, 735-752, doi: 10.1046/j.1365-246X.2003.01985.x.

Gucunski, N., and R. D. Woods, 1992, Numerical simulation of the SASW test: Soil Dynamics and Earthquake Engineering, 11, 213-227, doi: 10 1016/0267-7261(92)90036-D

Ikeda, T., and T. Matsuoka, 2013, Computation of Rayleigh waves on transversely isotropic media by the reduced delta matrix method: Bulletin of the Seismological Society of America, 103, 2083-2093, doi: 10.1785/ 0120120207

Ikeda, T., T. Tsuji, and T. Matsuoka, 2013, Window-controlled CMP crosscorrelation analysis for surface waves in laterally heterogeneous media: Geophysics, 78, no. 6, EN95-EN105, doi: 10.1190/geo20130010.1.

Ivanov, J., R. D. Miller, S. Peterie, C. Zeng, J. Xia, and T. Schwenk, 2011, Multi-channel analysis of surface waves (MASW) of models with high shear-wave velocity contrast: 81st Annual International Meeting, SEG, Expanded Abstracts, 1384-1390.

Ivanov, J., R. D. Miller, and G. Tsoflias, 2008, Some practical aspects of MASW analysis and processing: Presented at the Symposium on the Application of Geophysics to Engineering and Environmental Problems, 1186-1198.

Ivanov, J., C. B. Park, R. D. Miller, and J. H. Xia, 2006, Analyzing and filtering surface-wave energy by muting shot gathers: Journal of Environmental and Engineering Geophysics, 10, 307-322, doi: 10.2113/JEEG10 3.307.

Ivanov, J., T. J. Schwenk, R. D. Miller, and S. Peterie, 2013, Dispersioncurve imaging nonuniqueness studies from multi-channel analysis of surface waves (MASW) using synthetic seismic data: 83rd Annual International Meeting, SEG, Expanded Abstracts, 1794-1800.

Kato, A., S. Onozuka, and T. Nakayama, 2008, Elastic property changes in a bitumen reservoir during steam injection: The Leading Edge, 27, 1124 1131, doi: 10.1190/1.2978974.

Lin, C.-P., C.-C. Chang, and T.-S. Chang, 2004, The use of MASW method in the assessment of soil liquefaction potential: Soil Dynamics Earthquake Engineering, 24, 689-698.

Luo, Y., J. Xia, J. Liu, Q. Liu, and S. Xu, 2007, Joint inversion of high-frequency surface waves with fundamental and higher modes: Journal of Applied Geophysics, 62, 375-384, doi: 10.1016/j.jappgeo.2007.02.004.

Maraschini, M., F. E. Ernst, S. Foti, and L. V. Socco, 2010, A new misfit function for multimodal inversion of surface waves: Geophysics, 75, no. 4, G31-G43, doi: 10.1190/1.3436539.

Miller, R. D., J. Xia, C. B. Park, and J. M. Ivanov, 1999, Multichannel analysis of surface waves to map bedrock: The Leading Edge, 18, 1392-1396, doi: $10.1190 / 1.1438226$.

Mougenot, D., 2006, Toward the low frequencies: Land and marine equipment: Presented at the 6th International Conference \& Exposition on Petroleum Geophysics, 126-130.

Nakayama, T., A. Takahashi, L. Skinner, and A. Kato, 2008, Monitoring an oil-sands reservoir in northwest Alberta using time-lapse 3D seismic and 3D P-SV converted-wave data: The Leading Edge, 27, 1158-1175, doi: 10.1190/1.2978979.

O’Neill, A., M. Dentith, and R. List, 2003, Full-waveform P-SV reflectivity inversion of surface waves for shallow engineering applications: Exploration Geophysics, 34, 158-173.

Park, C. B., R. D. Miller, and J. Xia, 1998, Imaging dispersion curves of surface waves on multi-channel record: 68th Annual International Meeting, SEG, Expanded Abstracts, 1377-1380.

Park, C. B., R. D. Miller, and J. Xia, 1999, Multichannel analysis of surface waves (MASW): Geophysics, 64, 800-808, doi: 10.1190/1.1444590.

Ryden, N., and M. J. S. Lowe, 2004, Guided wave propagation in three-layer pavement structures: Journal of the Acoustical Society of America, 116, 2902-2913, doi: 10.1121/1.1808223.

Ryden, N., and C. B. Park, 2006, Fast simulated annealing inversion of surface waves on pavement using phase-velocity spectra: Geophysics, 71 no. 4, R49-R58, doi: 10.1190/1.2204964.

Saito, M., 1988, DISPER80: A subroutine package for calculation of seismic normal-mode solution, in D. J. Doorbos, ed., Seismological algorithms: Academic Press.

Saito, M., and H. Kabasawa, 1993, Computation of reflectivity and surface wave dispersion curves for layered media: 2 . Rayleigh wave calculations (in Japanese): Butsuri-tansa, 46, 283-298

Socco, L. V., D. Boiero, M. Maraschini, M. Vanneste, C. Madshus, H. Westerdahl, K. Duffaut, and E. Skomedal, 2011, On the use of the Norwegian Geotechnical Institute's prototype seabed-coupled shear wave vibrator for 
shallow soil characterization - II. Joint inversion of multimodal Love and Scholte surface waves: Geophysical Journal International, 185, 237-252, doi: 10.1111/j.1365-246X.2011.04961.x.

Socco, L. V., S. Foti, and D. Boiero, 2010, Surface-wave analysis for building near-surface velocity models - Established approaches and new perspectives: Geophysics, 75, 75A83-75A102, doi: 10.1190/1.3479491.

Socco, L. V., and C. Strobbia, 2004, Surface-wave method for near-surface characterization: A tutorial: Near Surface Geophysics, 2, 165-185.

Strobbia, C., A. E. Emam, J. Al-Genai, and J. Roth, 2010, Rayleigh wave inversion for the near-surface characterization of shallow targets in a heavy oil field in Kuwait: First Break, 28, 103-109.

Strobbia, C., A. Laake, P. Vermeer, and A. Glushchenko, 2011, Surface waves: Use them then lose them. Surface-wave analysis, inversion and attenuation in land reflection seismic surveying: Near Surface Geophysics, 9, 503-514, doi: 10.3997/1873-0604.2011022.
Takahashi, A. 2010, Oil-sands reservoir characterization for optimization of field development, in S. Chopra, L. Lines, D. R. Schmitt, and M. Batzle, eds., Heavy oils: Reservoir characterization and production monitoring: SEG Geophysical Developments Series, 13, 227-234.

Tokimatsu, K., S. Tamura, and H. Kojima, 1992, Effects of multiple modes on Rayleigh wave dispersion characteristics: Journal of Geotechnical and Geoenvironmental Engineering, ASCE, 118, 1529-1543.

Tsuji, T., T. A. Johansen, B. O. Ruud, T. Ikeda, and T. Matsuoka, 2012, Surface-wave analysis for identifying unfrozen zones in subglacial sediments: Geophysics, 77, no. 3, EN17-EN27, doi: 10.1190/geo20110222.1.

Xia, J., R. D. Miller, C. B. Park, and G. Tian, 2003, Inversion of high frequency surface waves with fundamental and higher modes: Journal of Applied Geophysics, 52, 45-57, doi: 10.1016/S0926-9851(02)00239-2. 